need for assuming some law of angular distribution of scattered light which may be far from the truth. The visibility of an object which is not too small depends on the ratio of its surface brightness, as seen, to that of the surrounding field. This ratio must differ from unity by an amount which is almost independent of the absolute brightness (provided this is not too small), and may. according to Ruedy, be taken as 2 per cent. The chief obstacles to visibility are $(a)$ the reduction of the ratio of brightnesses by the addition to both of the daylight scattered by the intervening medium; $(b)$ the reduction in absolute difference of brightness by extinction in the intervening medium, including the mixing of light from the object and field by scattering; (c) the possible reduction of illumination to such a degree that increased ratios are needed to ensure detection.

The extinction of red light reduces all objects to a green or bluish green hue a few metres under water. At greater depths, when the eye is dark-adapted, colour sensation is lost; visibility is, however, helped by the fact that the maximum sensitivity is then close to the wave-length which is best transmitted by sea-water.

It is evident that the distance at which an object is visible must vary greatly with its direction. A light-toned object, such as the Secchi disk, is probably most visible against the dark background of the water directly below the observer, although here the intervening scattering particles are more strongly illuminated than the object. A dark-toned object should be more visible in a horizontal direction, while the visibility of any object overhead must largely be governed by the degree of small-angle scattering. The presence of direct sunlight would introduce azimuthal asymmetry and further complicate the problem.

It would seem then that, pending further increase in our knowledge of the angular distribution of scattered light and of the resultant distribution of field brightness, it is difficult to estimate with certainty the visibility of a given object from measurements of light penetration alone.

'National Research Council of Canada, No. 1061.
${ }^{2}$ Ann. d'Inst. Ocean., 19, Fasc. 4 (1939).

${ }^{3}$ J. Mar. Biol. A880c., 24, 271 (1940).

\section{THE FIGURE OF THE EARTH}

A GEOPHYSICAL Discussion was held on August 14 in the rooms of the Royal Astronomical Society, when the subject dealt with was "The Figure of the Earth".

Sir Gerald Lenox-Conyngham opened the discussion with a statement of the values obtained by Airy, Everest, Bessel, Clarke and Hayford for the semi-axis major of the earth and for the ellipticity of figure. In attempts to eliminate so far as possible the effects of local deflexions of the plumb-line, Airy avoided arcs measured in mountainous country, while Everest used only the longest arcs available. The fundamentally important question arises as to whether these local deflexions may be treated as random errors ; if so, it is appropriate to use the method of minimum squares in the reduction of the observation. This was the method employed by Bessel, and the fact that Airy did not use it suggests that he did not regard the deflexions as random.

An important development began with Waugh's suggestion to Archdeacon J. H. Pratt, of Calcutta, that he should calculate the gravitational pull of the Himalayas at various stations of the triangulation, with the view of correcting the observed latitudes. The calculated attractions were greatly in excess of those indicated by the observations, and led to Pratt's well-known theory of mountain compensation, and Airy's equally famous hypothesis of the "roots of the mountains". It seemed, then, that at moderate or at great distances the attraction of the mountains was for the most part compensated, and that deflexions observed well away from mountain ranges might be ascribed mainly to local causes, which would have a random effect on observations made at an evenly distributed set of stations.

Burrard's discussion of the accumulated Indian data in 1901 led to an important modification of this view. He found that the local deflexions showed systematic trends in directions parallel to the Himalayas; nor could their amounts be attributed to any system of mountains and their compensation. It was, therefore, incorrect to suppose that mountain attraction did not extend to any great distance, or to treat deflexions as random errors.

In 1909 Hayford published the results of his determination of the earth's axis and figure by a new method. Accepting mountain compensation as normally complete and assuming that below a depth of $113 \mathrm{~km}$. there is hydrostatic equilibrium, he computed the effect of all topographical irregularities at each station of observation, and applied the result of the computation as a correction to the observed latitude, longitude or azimuth, as the case might be. There is, therefore, a difference between the smoothed geoid that Hayford was endeavouring to fit and that which had been dealt with in earlier determinations. Hay. ford, moreover, uses the data of the United States of America only; if a similar determination covering Australia could be made, the results would be of the highest interest.

Dr. R. Stoneley indicated how closely geodesy, astronomy and geophysics are interconnected. The connexion between the gravitational field of the earth and its geometrical form is noticed as soon as one uses a plumbline or spirit-level. It is natural to use as a reference surface a gravitational equipoten. tial, and the equipotential that coincides with mean sea-level is termed the 'geoid'; since this is not a simple geometrical surface, it is convenient to use for reference a spheroid decided upon by international agreement. In a corresponding way gravity may be compared with a standard gravity formula. The relationship between trigonornetrical work and the intensity of gravity is seen in Clairaut's well-known formula; the variation of gravity with latitude determines the ellipticity of the figure of the earth. It is assumed that the ocean is free from shearing stress, but no assumption is made concerning the distribution of density within the earth.

A theorem due to George Green shows that a knowledge of the external gravitational field does not determine uniquely the density distribution within the earth. None the less, some information of a general character is forthcoming from the theory of the earth's attraction; if $A, A, C$ are the principal moments of inertia, $M$ the mass, $a$ the mean radius of the earth, and $m$ the ratio of centrifugal force to gravity at the equator, then the ellipticity $(\varepsilon)$ of figure is given by $J=3(C-A) / 2 M a^{2}=\varepsilon-\frac{1}{2} m$. The number $J$ can be found independently from the monthly term in the moon's latitude. The 'dynamical 
compression' $(C-A) / C$ is known from the period of the precession of the equinoxes, and with the pre. ceding formula leads to the value $0 \cdot 334$ for $C / M a^{2}$, whereas in a homogeneous earth the value would be $0 \cdot 4$. Thus, the earth must on the whole be centrally condensed. Likewise, a knowledge of $J$ and $m$ determines $\varepsilon$ with great accuracy.

A further advance can be made if it is assumed that the earth is to a near approximation in hydrostatic equilibrium for forces of long period. The work of Callandreau and of Sir George Darwin then shows that, given $\varepsilon, m$, and the dynamical compression, the value of $C$ can be found; the calculation indeed is so insensitive to the assumed law of density that the known ellipticity cannot be used to test a law of density that gives the correct value of $C$.

In spite of the restrictions imposed by Green's theorem, one can use observations of gravity over a restricted area to derive some information about the density distribution a little way below the surface. Some of the mathematically possible distributions involve negative masses, and in practice other methods, such as seismic exploration, are available to check gravitational measurements. Within these limitations, much scientifically and commercially useful information may be obtained.

The Astronomer Royal pointed out that the addition of the Callandreau-Darwin theory to the work of Clairaut brings in the assumption that the earth is in hydrostatic equilibrium throughout. Since the actual land surface is not a gravitational equipotential, the validity of this extra assumption requires critical examination. The justification is due to de Sitter, who assumed the earth to be in hydrostatic equilibrium from the centre up to a surface which he named the 'isostatic surface'; the undisturbed surface of the ocean was taken to be part of the geoid, and between these two surfaces the equipotential surfaces would not necessarily be spheroidal, nor would they necessarily be surfaces of equal density. If the hydrostatic condition were satisfied throughout, the upper surface of the (ideal) body representing the earth would be a surface which de Sitter calls the 'ideal surface'. The spheroid which is the best fit to the geoid he calls the 'normal surface'.

If the outer potential of a body which is symmetrical both with regard to an axis of rotation and to an equatorial plane is expanded in a power series in $1 / r$ and it is assumed that the surface which is an equipotential surface is nearly a spheroid, the boundary conditions give for a body of the size, mass and angular velocity of the earth the relations

$$
J=\varepsilon-0.0017287 ; \beta=0.0086559-\varepsilon,
$$

where $\beta$ is the coeflicient of the term in $\sin ^{2} \phi^{\prime}$ in the formula for gravity at a point of tho earth's surface ; as already mentioned, the internal constitution is not involved in these formula. If we write $H$ for the dynamical compression $(C-A) / C$, we obtain the equation $J / H=q=f(\varepsilon)$, which is applicable to the ideal surface, but not necessarily to the normal surface.

The definition of the normal surface as the spheroid of best fit to the geoid implies that in the development of both surfaces in a series of spherical harmonics the coefficients of the harmonics of degrees $0,2,4$ are the same. It follows that for the normal surface $J$ is the same as for the geoid. Writing for the idcal surface $J_{1}=q_{1} H_{1}$, where $q_{1}$ is a function of $\varepsilon$, and for the actual carth $J=q H$, where $q=3 C^{\gamma} / 2 M a^{2}$, we have
$J=J_{1}$, and it remains to find the difference between $q$ and $q_{1}$. The computation made by de Sitter assumed that the actual earth differs from an ideal hydrostatic earth in having an excess of mass in the continents and a deficiency in the oceans ; these mass anomalies are supposed to be isostatically compensated by a 'compensation' which is uniformly dis. tributed throughout the material above the isostatic surface. If the sulfix 1 refers to the ideal surface the results become :

$\left(C-C_{1}\right) / C_{1}=-0 \cdot 000,000,34 ; H-H_{1}=-0 \cdot 030,000,85$

$q-q_{1}=-0.000,000,03 ; \varepsilon^{-1}-\varepsilon_{1}^{-1}=0.04$,

so that the difference in the ellipticities is entirely negligible. If there were no isostatic compensation the difference in the values of $\varepsilon$ would have been about two units.

Astronomical theory gives $J=\varepsilon-0.0017287$ $=q / P\left(A+B \mu^{\prime}\right)$, where $P$ is the precessional con. stant, $A$ and $B$ are known with great accuracy from theory, and $\mu^{\prime}$ depends on the ratio of the masses of the earth and moon. If, then, the assumption is conceded that the earth below the isostatic surface is in hydrostatic equilibrium (and there is evidence that this assumption is not strictly correct), this method gives the value of the compression with a smaller uncertainty than any other method.

Dr. E. C. Bullard discussed how far the values of $1 / \varepsilon$ given by various methods agree with de Sitter's result. These values are:

$\begin{array}{ll}\text { Motion of lunar perigee } & 296 \cdot 6 \pm 1 \cdot 2 \\ \text { Motion of lunar node } & 296 \cdot 0 \pm 1 \cdot 2 \\ \text { Motion of moon in latitude } & 297 \cdot 3 \pm 0 \cdot 9 \\ \text { Gravity observations } & 296 \cdot 6 \pm 0 \cdot 5 \\ \text { Lunar parallax } & 294 \cdot 7 \pm 1 \cdot 4 \\ \text { Triangulation (Hayford) } & 297 \cdot 0 \pm 0 \cdot 5 \\ \text { Precession } & 297 \cdot 24 \pm 0 \cdot 044 \\ \text { Nutation } & 297 \cdot 61 \pm 0 \cdot 12\end{array}$

Of these determinations, the Iunar parallax method depends on measurements at Greenwich and the Cape only, and may be suspected of serious systematic error. The mean of the first three is $296 \cdot 7 \pm 0 \cdot 6$; this astronomical determination, combined with that from gravity observations, gives $296 \cdot 6 \pm 0 \cdot 4(A)$. Accepting de Sitter's justification of the use of the precessional method, which involves the assumption of hydrostatic equilibrium, that method is capable of high precision, and the result differs from the preceding result $(A)$ by $0 \cdot 6 \pm 0 \cdot 4$. This difference may not be significant, but if the difference is real, it implies stress differences of the order of $2 \times 10^{7}$ dynes $/$ cm. ${ }^{2}$, which are by no means unlikely.

Dr. H. Jeffreys recalled his attempt in 1941 to represent the observations of gravity over the earth's surface in terms of spherical surface harmonics. The problem is not statistically simple, for the existing observations are by no means uniformly distributed over the globe. In that determination, apart from the main ellipticity term, none of the coefficients was certain even as to sign. To carry through a least squares solution with twenty unknowns would involve very heavy computation, and therefore Dr. Jeffreys had now proceeded by a method of successive approximation. As a first step the normal equations were regarded as orthogonal, and only terms large compared with their standard error were retained. Dopartures from the international gravity formula were represented by

$g-g_{0}=\Sigma\left(a_{m, n} P_{n}^{m} \cos m \lambda\right)+\Sigma\left(b_{m, n} P_{n} m \sin m \lambda\right)$. 
The coefficients given by the first approximation, with their standard errors, were $a_{22}=4 \cdot 8 \pm 1 \cdot 3$; $a_{31}=6 \cdot 2 \pm 2 \cdot 3 ; \quad a_{32}=2 \cdot 0 \pm 0 \cdot 6$, none of the others being as great as their standard errors. For a second solution he included $a_{00}$ and also $a_{20}$ (the main ellipticity term) and solved for the five coefficients by least squares. The result was : $a_{22}=4 \cdot 0 \pm 1 \cdot 4 ; a_{32}=$ $1 \cdot 3 \pm 0 \cdot 7 ; a_{31}=4 \cdot 2 \pm 2 \cdot 4 ; a_{00}=2 \cdot 5 \pm 1 \cdot 9 ; a_{20}=-6 \cdot 1 \pm$ 5.0 ; and the cosificient $b_{33}$ then had the value of $0 \cdot 46 \pm 0 \cdot 26$. The coefficient $a_{22}$ indicates that the 'ellipticity of the equator' is genuine, but that the uncertainty is greater than that given by Heiskanen.

Compensated inequalities that would account for the harmonics $a_{22}, a_{32}, a_{31}$ and $b_{33}$ would be of amplitudes $5 \cdot \mathbf{2}, \mathbf{1} \cdot \mathbf{9}, \mathbf{1} \cdot \mathbf{9}, \mathbf{2} \cdot \mathbf{2} \mathrm{km}$. respectively. Now Prey computed in 1922 coefficients which give the representation of the earth's figure by spherical harmonics up to degree 16, and from his results it follows that there is practically no relation between mean height and gravity anomaly for inequalities with horizontal ranges of about $30^{\circ}$. Thus, the hypothesis of complete compensation must be abandoned. To explain the distribution of gravity an internal interface seems to be required, the height of which has a negative correlation with that of the outer surface. We have, in fact, strong evidence that isostasy is not exact, and are led to something of the type of Glennie's 'crustal warping'. The value of the reciprocal of the ellipticity of the earth derived from this investigation is $296 \cdot 17$, with a standard error of $0 \cdot 68$.

\section{R. Stoneley.}

\section{OBITUARIES}

\section{Prof. F. E. Suess}

News has been received recently that Prof. Franz Eduard Suess, emeritus professor of geology in the University of Vienna, the son of Eduard Suess, died in Austria about a year ago, aged seventy-four. After many years on the Austrian Geological Survey, he occupied several academic posts before his appointment to the chair in Vienna in 1911. As an enthusiastic pupil of Becke, he was one of the first to make use of the petrology of metamorphism to interpret the history of rocks, a method he applied in grappling with problems of the crystalline massif of Moldanubia and studies of the deeper parts of mountain ranges where intrusions play an important part. Later, he integrated his opinions by enunciating a theory of mountain building through loading caused by the carriage of a moving 'creative mass' over a passive foreland. The ranges as they exist to-day result from the erosion of this structure. Fragments of the load left by chance can be distinguished from the loaded under-ground by the different grade of metamorphism they display, and a passage can be traced from the loaded to the unloaded part of the old foreland. It follows that the occurrence of a mass of crystalline schists in a mountain system is no evidence that they are older than fossiliferous sediments not far away in the unloaded region. Indeed, the sedimentary rocks in the 'creative mass' may be as old as the schists and gneisses formed below them. He regarded the Caledonian and Variscan ranges of Europe, Asia and America as types of this orogenesis.

Suess was less prolific than his father, but he displayed an enviable erudition in contributions to a host of geological subjects. His lectures in Vienna were popular, partly on account of his masterly use of the
German language, and partly because they covered the geological globe in a well-balanced conspectus supported by a background of documented facts. $\mathrm{He}$ was particularly attracted to Britain, where his father was born, and visited the Highlands more than once to compare their architecture with that of the Caledonian masses he knew so thoroughly near the Danube. He contributed papers in English on the thorny problems of the structures of the Scottish Highlands, and they provide a welcome variety in a rather mystic literature.

Though somewhat crippled in later years, Suess was surprisingly active, and in pursuit of his science he would walk long days amidst the woods of the Wiener Wald or on his own particular ground not far from the Danube, over the Scottish moors in their less pleasant moods, or brave the winter frosts of the Bushveldt and sleep with only a cloak between him and the stars at an age when such expeditions would dismay most men.

Suess was an LL.D. of the University of Glasgow and had a large circle of friends in Great Britain. $\mathrm{H}_{\Theta}$ had a discriminating taste in art and music, in both of which he was no mean performer. The former rendered his field notebooks models of their kind as his skilful, truthful pen recorded pictorial evidence unbiased by theoretical distortion and furnished illustrations of field facts in a way that few can hope to do even with the aid of a camera. In the latter, his piano was near his study and upon it he would discourse in a most versatile fashion. Like many an Austrian he loved Mozart and Schubert, Beethoven and Wagner, but besides he was tremendously attracted by the unexpected harmonies of Richard Strauss. He had an unusual knowledge and appreciation of the whimsicalities of Gilbert and Sullivan. $\mathrm{He}$ attracted around him a large circle of students and friends who used to meet once a week in one of the parks near the Danube during each summer. To those who knew him he was ever genial and as a man hospitable to a fault.

\section{J. V. Harrison.}

\section{Mr. E. A. Nahum}

MR. E. A. Nahum, a research worker in the Cavendish Laboratory, was killed in Cambridge during a recent air raid.

Nahum, who belonged to a Manchester family, was educated at Clifton College and at Pembroke College, Cambridge, to which he went with an Open Exhibition in 1936. He read for the Natural Sciences Tripos and obtained a first class in Part I and in Part II (Physics). He returned to Cambridge with a Goldsmith senior studentship in 1940 and began work on the cyclotron. The Cambridge cyclotron had not long been installed, and as most of the senior nuclear physicists were away, it says much for the young research workers that they not only kept it in running order but also obtained material for four very comprehensive papers on the deuteron bombardment of the heavy elements. In the last two of these, with R. S. Krishnan, which appeared in a very recent number of the Proceedings of the Royal Society, the first quantitative measurements were made of the reaction yields, which made possible detailed comparison of experiment with results predicted by the present theory of the interactions of deuterons with heavy nuclei.

Nahum was known to many as a young scientific worker of great promise, but students throughout 